\title{
Lord, deliver us from pain
}

\author{
Francis Veyckemans and Daniel Rodenstein
}

Affiliations: Depts of Anaesthesiology and Pneumology, Cliniques Universitaires Saint-Luc, Université catholique de Louvain, Brussels, Belgium.

Correspondence: D. Rodenstein: Service de pneumologie, Cliniques universitaires Saint-Luc, Av. Hippocrate 10, 1200 Brussels, Belgium. E-mail: Daniel.rodensteinduclouvain.be

0 @ERSpublications Respiratory depressant effects necessitate some degree of caution when prescribing buprenorphine or other opioids http://ow.ly/kRCqp

Pain is a word every human being can understand. Except those with congenital insensitivity to pain there is probably no human being that has completely escaped the perception of pain. Pain is an unpleasant feeling related to a physical aggression compromising the integrity of the body. It also has an emotional component that may be weak or strong. The fear of pain may be as disturbing as pain itself, as many a torturer knows and uses.

There are experts on pain in the medical field. Their aim is not to cause pain, but to understand and alleviate it. The International Association for the Study of Pain has modified the simple aforementioned definition we can all understand, by specifying that pain can be felt in the face not only of an actual tissue damage, but also in the face of a "potential damage, or described in terms of such damage" [1], whatever this may mean for the layman.

If we try to put it in simple everyday words, this definition means that the pain someone experiences from actual physical damage to some body tissue is of the same nature as pain someone feels without any physical damage being actually visible, or without the possible immediate risk of such damage being inflicted. It pertains to the same category, produces the same consequences and requires a similar attitude.

We are not pain experts (except for the one we experience ourselves from time to time and the one we frequently observe in our patients), but we have to admit feeling some reluctance at accepting this extended definition of pain. We are inclined to consider that pain feelings combined with tissue damage are more biologically sound than pain felt, but unrelated to actual tissue aggressions. We would easily describe the former as "physiological pain", the latter (e.g. after tissue damage has healed) as "unphysiological pain" or neuropathic pain or, in some cases, "psychopathological pain".

This being said (and it is important to consider our background for what will follow), this issue of the European Respiratory Journal includes a very interesting research study by FARNEY et al. [2] showing, in very short summary, that buprenorphine has a respiratory depressant effect during sleep. The respiratory depressant effects of morphine are well known, although frequently overlooked and underappreciated with dreadful consequences [3]. Morphine in appropriate doses reduces the respiratory frequency and tidal volume, induces periodic breathing in the form of Biot's respiration (ataxic breathing), leads to apnoeas and hypoventilation and can eventually lead to respiratory arrest and death. Morphine is, therefore, frequently used as part of the standard cocktail in terminal patients, irrespective of the presence of pain.

Morphine is potentially addictive, and many consumers outside the field of pain treatment end up with an extreme form of physical and psychological dependency [4]. This can be dealt with through various types of therapies, including replacing morphine with less toxic products and so "cleaning" the addiction to the more toxic substance. FARNEY et al. [2] have developed an inpatient care programme using buprenorphine,

Received: Jan 102013 | Accepted: Jan 112013

Conflict of interest: None declared.

Copyright (CERS 2013 
alone or combined with naloxone, which includes a careful scrutiny of the respiratory aspects of its administration at the most vulnerable time, i.e. during sleep. They performed full-night polysomnography in their patients whenever possible, depending on sleep laboratory availability. This was carried out after the initial treatment phase, just before the stabilisation phase, once withdrawal symptoms had decreased. Their main findings were that respiration is frequently abnormal, with central apnoeas, ataxic breathing and hypoxaemia as the main events. These breathing abnormalities seemed to be unrelated to previous smoking history, to buprenorphine dosage or associated drugs (patients also received benzodiazepines and neuroleptics). Whether these abnormalities are a residual trace of the long-term exposure of the different opioid receptors to high doses of morphine or a specific effect of buprenorphine is not known. Moreover, these patients were not in pain and clinicians know that residual pain seems to protect against respiratory depression, at least during wakefulness. FARNEY et al. [2] did not assess the clinical consequences of their findings (i.e. an eventual increase in deaths related to buprenorphine use), but their study provides a physiopathological link leading to some degree of necessary caution whenever buprenorphine (and possibly any other opioid in its present day multiple formulations) are prescribed in opioid-naive as well as in longterm users of morphine.

Buprenorphine is generally believed to be safe, although its respiratory depressant effects are known to exist [5]. However, its widespread use (in Belgium, its use increased ten-fold from 1997 to 2011) to treat chronic pain makes any respiratory depressant effect potentially dangerous. Especially given that there is a known association between opioid use, regardless of indication, and unintentional opioid-related deaths [3].

The campaigns aimed at increasing awareness of pain, especially chronic pain, as an important symptom, have fuelled the impressive increase in opioids prescription by the medical field. The study by FARNEY et al. [2] is a welcome reminder to everyone prescribing opioids.

\section{References}

IASP Task Force on Taxonomy. Merskey H, Bogduk N, eds. Classification of Chronic Pain. 2nd Edn. Seattle, IASP Press, 1994.

2 Farney RJ, McDonald AM, Boyle KM, et al. Sleep disordered breathing in patients receiving therapy with buprenorphine/naloxone. Eur Respir J 2013; 42: 394-403.

Okie S. A flood of opioids, a rising tide of deaths. N Engl J Med 2010; 363: 1981-1985.

Boyert EW. Management of opiod analgesic overdose. N Engl J Med 2012; 367: 146-155.

Pattinson KTS. Opioids and the control of respiration. Br J Anaesth 2008; 100: 747-758. 